\title{
Smell reduction and disinfection of textile materials by dielectric barrier discharges
}

\author{
Siegfried Müller ${ }^{1 \star}$, Rolf-Jürgen Zahn ${ }^{1}$, Torsten Koburger², Klaus-Dieter Weltmann ${ }^{1}$ \\ ${ }^{1}$ Leibnitz-Institut für Plasmaforschung und Technologie e.V., Greifswald, Germany; *Corresponding Author: muellers@inp-greifswald.de \\ ${ }^{2}$ Hygiene Nord GmbH, Greifswald, Germany
}

Received 10 May 2010; revised 18 June 2010; accepted 25 June 2010.

\begin{abstract}
In this paper we present investigations of textile cleaning of cotton fabrics with respect to both, the smell reduction and the disinfection of textile materials. Normal pressure plasma sources on the base of dielectric barrier discharge (DBD) were used for the purification and disinfection of textiles. For gaseous odour components which stick to clothing the results have shown that one can reach an uncritical odour threshold. In the case of disinfection a significant reduction of microorganism population in some of the samples could be noted. In particular a high reactivity is reached, while in parallel with a radiation by ultraviolet light ozone is activated.
\end{abstract}

Keywords: Dielectric Barrier Discharge; Odour Reduction; Ozone Activation; Disinfection and Sterilization; Pollution Control

\section{INTRODUCTION}

The purification and disinfection of textiles is linked with the use of water and diverse cleaning agents. This causes a considerable consumption of our water resources and leads to water charges. Water is becoming an increasingly scarce resource so that there is an urgent need to reduce the amount of water and energy used for washing clothes.

In this contribution we present investigations of textile cleaning and of disinfection by using plasma on the base of DBD. DBDs are widely used as a non-thermal plasma (NTP) technique in many plasma technological applications. Examples for NTP applications are air-pollution control systems, soot decomposition, destruction of microorganisms, and water treatment [1-5]. Fur-ther, a lot of other plasma processing applications like surface cleaning, modification and functionalization are under investigation [6].
In NTP's temperature differs substantially between the electrons and other particles (ions, atoms, molecules). The electric energy is supplied only to charged particles, mainly electrons $(\mathrm{T} \approx 10.000 \mathrm{~K}$ ), whereas the neutral gas molecules remain almost cold (nearly room temperature). In this case an NTP also refers to as non-equilibrium plasma or cold plasma.

A strong electric field causes an ionization of the working gas and the formation of radicals and ions. These are highly reactive species, and they are the basis for various plasma chemical reactions.

Besides the above applications it was demonstrated that one can eliminate or reduce odours from waste air in the process of fabricating French frees by special DBDconfigurations [7]. The experiments showed a correlation of odour strength with odourous compounds such as volatile organic substances (VOCs). The VOCs were identified mainly as aldehydes by using thermal desorption GC/MS. This was one background for the question of textile cleaning concerning odour control like in the case of airborne waste.

On the other hand microbial contaminations become important in a lot of fields related to textile cleaning. As it is known sterilization can be achieved by chemical and/or physical treatment, such as heat, chemical solutions, gases and radiation. But these methods have numerous detrimental effects on the environment and substrates. In the last few decades, medicine and food industries have conducted research to adopt new sterilization methods.

The sterilization with NTP arose as one of the successful solutions destroying microorganisms. Sterilization agents of plasma are radiation and particles (electrons, ions, radicals) [8-16].

Today, NTP can be generated in wide range of pressures and with various means, such as the use of microwave, RF, pulsed, ac and dc power sources. Several device geometries and electrode configurations have been used. Among these, the DBD, the resistive barrier discharge and the atmospheric pressure plasma jet have been 
especially researched in the past few years [12].

The use of special plasma methods in the textile industry is also well-known. The spectra of applications of plasma technologies ranges from the functionalization of fibre surfaces to the production of special layers. Surface properties like wettability, refractability, colourability and printability or surface hardness can be influenced without changing the bulk qualities of the substrate $[17,18]$.

The purpose of this investigation was to test the cleaning of cotton fabrics with respect to the smell reduction and the disinfection using different DBD plasma configurations.

\section{REACTOR CONFIGURATIONS AND EXPERIMENTAL SET UP}

\subsection{Discharge Configurations}

In the process, textiles were exposed to the plasma in different devices and by special methods. For the experiments we used different plasma reactor constructions on the basis of DBD configurations, partly described in $[7,19]$. In principle a DBD consists of two electrodes with at least one insulating layer (dielectric barrier) in between. The DBD can be operated in a wide pressure range (mbar-range up to atmospheric pressure range); the electrode distance is in the range of tenth of $\mathrm{mm}$ up to some mm. Generally the DBE can be sustained with sine wave or square wave voltages in the frequency range of some $\mathrm{Hz}$ up to some $100 \mathrm{kHz}$.

Three basic DBD configurations have been used. One configuration consists of a flat arranged metallic bottom electrode, a dielectric on the top of the bottom electrode, a discharge gap and a second electrode. The dielectric barrier is formed by a composite material of mica with a thickness of $1 \mathrm{~mm}$. In case of odour treatment the size of the top electrode equals the dimension of textile patches $(120 \mathrm{~mm} \times 75 \mathrm{~mm})$. In the experiments square wave voltages with positive or negative polarity have been used.

Besides the conventional configuration a stacked DBD system was used. The special features of this reactor configuration are structured electrodes arranged in a compact pattern; the structured electrodes alternate with dielectric flat disks. An exploded view of such a stack configuration is given in [7]. In addition, spacers are mounted between the mesh electrodes and the dielectrics. The structured electrode consists of woven wires of stainless steel with a wire thickness of $0.5 \mathrm{~mm}$ and a mesh size of $0.8 \mathrm{~mm}$. The dielectric barrier is formed again by a composite material of mica. The stack is sealed on two opposite sides and enables the processing gas to flow through the configuration. The complete sys- tem is mounted in a casing. The stack planes of wire mesh are put alternately on high voltage potential or ground potential alternately. At the reactor outlet a pipe was mounted which serves as flow path and treatment space.

Additionally, in some experiments, a low-pressure mercury lamp containing a quartz window was used. The lamp was positioned at a side wall opening of the pipe and radiates a part of the treatment space.

The third configuration contains a surface discharge DBD (SD-DBD) with structured electrodes mounted at the top of a casing. In the considered assembly a woven wire electrode is positioned on top of a dielectric flat disk while the second one is on its reverse side. The types of wire electrodes and dielectric barriers are the same as in case of stacked DBD.

Furthermore we constructed a setup where an extraction electrode in front of the SD-DBD was mounted. This way an ion extraction from the discharge region can be performed. The extraction electrode serves also as holder for the textiles. The SD-DBD was sustained by square wave or sine wave pulse voltages. The plasma is ignited at the side wall in a small part of the reactor, and the textiles are not in touch with the plasma part. Positive or negative ions are extracted from the discharge side by the given potential difference and penetrate the textiles.

The electrical parameters of all three discharge configurations were determined by a digital oscilloscope in the usual way.

For the comparison of the microbiological activity we used finally a commercial air purifier which is based on a corona discharge configuration. In the product specification more than $10^{6}$ negative ions $/ \mathrm{cm}^{3}$ were advertised.

\subsection{Odour and Microbiological Procedures}

For odour control the cotton fabric was situated within time duration of 12 hours in the exhaust pipe of a cooker hood from a food restaurant. For the different experiments we prepared patches of $120 \mathrm{~mm} \times 75 \mathrm{~mm}$. In our experiments the olfactometry was used for the smell analysis. In order to realize this, the different patches were put in bags of inert material with a volume of approx. 8 litres. For the determination of the basic level at smells, further samples of the untreated and unloaded air were taken from the surroundings.

The microbiological tests were carried out using Escherichia coli K12 (NCTC 10538) obtained from the German Collection of Microorganisms and Cell Cultures (DSMZ) as lyophilized cultures. The test organisms were grown and cultivated in accordance with the DIN EN 12353 "Chemical disinfectants and antisepticsPreservation of test organisms used for the determination of bactericidal and fungicidal activity". Small pieces (30 
$\mathrm{mm} \times 30 \mathrm{~mm}$ ) of cotton cloth (DIN 53919) were used as reference fabric and germ carriers. They were prepared by rinsing them in distilled water, followed by steam sterilization. After that the small pieces of cotton cloth were contaminated by submerging them in a suspension of E. coli. After a drying time of $3 \mathrm{~h}$, they were either stored away aseptically or exposed to the plasma in the reactor right away. After the exposure, the number of surviving bacteria was individually determined for each germ carrier by vigorously vortexing it in test tubes containing $5 \mathrm{ml}$ nutrient broth (TSB) and glass beads and transferring defined amounts and dilutions of the resulting solution, as well as the germ carrier, to agar plates (TSA). After an incubation time of $48 \mathrm{~h}$ at $36^{\circ} \mathrm{C}$ bacterial colonies were counted, the number of surviving bacteria was calculated and compared to that obtained from non-plasma treated germ carriers.

\section{RESULTS AND DISCUSSION}

For the removing of smells from textiles three discharge configurations as described above were used. With different installations the impact of different effects was evaluated. Figure 1 shows the comparison of these versions after the plasma treatment with air as carrier gas.

The results show a clear degradation of odour strength by plasma application. Compared to the untreated sample this upshot occurred in all three constellations. By using plasma methods reactions by long living species like ozone are predominantly pronounced.

In the case of ion extraction method the textiles are exposed for 1 hour to the ions coming from the plasma electrode in a distance of $20 \mathrm{~cm}$. The discharge was sustained by negative pulse voltages with amplitudes of 7.5 $\mathrm{kV}$ and frequencies of $5 \mathrm{kHz}$. In this case the reduction of odour strength in addition is based on the effect of negative ions.

With the direct treatment of the patches in the discharge

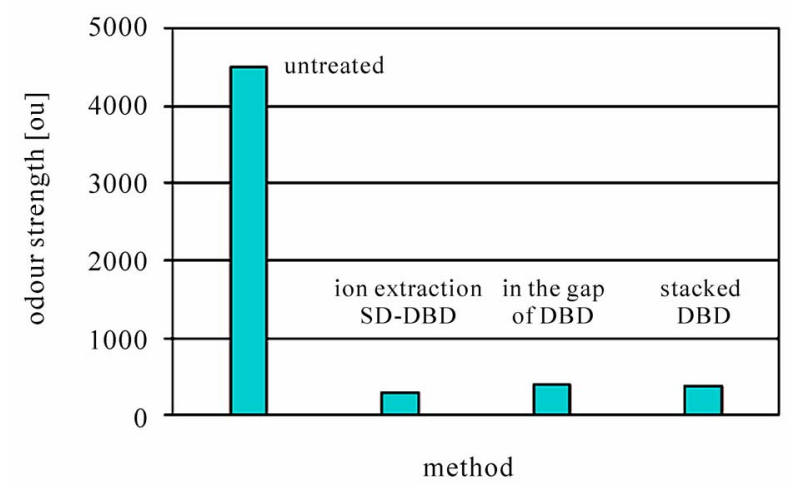

Figure 1. Odour strength after different plasma treatment procedures. gap similar results could be reached under comparable discharge conditions (treatment time of 6 minutes).

In case of stacked-SD-DBD the textile material is arranged on the exhaust-side of the reactor. Reactions by ozone which is streaming out of the reactor become especially dominant.

As another procedure in the microbial analysis an activation of oxygen was tested. Figure 2 shows the experimental set up. Ozone generated in the discharge was exposed to UV radiation emitted by a $\mathrm{Hg}$-low pressure discharge lamp.

In Figure 3 this procedure schematically is demonstrated. In the experiments we applied an interference filter on demand to filter out the $254 \mathrm{~nm}$ resonance line.

The short wavelength resonance line $(185 \mathrm{~nm})$ leads to the formation of ozone by cracking oxygen molecules, but the line at $254 \mathrm{~nm}$ decomposes the ozone whereas highly reactive oxygen radicals (activated oxygen) are generated.

Figure 4 shows the effect of radiation of ozone at 254 $\mathrm{nm}$. In this experiment ozone was generated with a commercial ozonizer and the concentration has been measured, using UV absorption spectroscopy. The experiments took place in a box with a total volume of 100 litres.

As clearly recognizable, a quick decrease of the ozone concentration occurs by switching on the UV radiation ( $254 \mathrm{~nm}$ line) because of the following decomposition of the ozone. After switching off the lamp a higher ozone

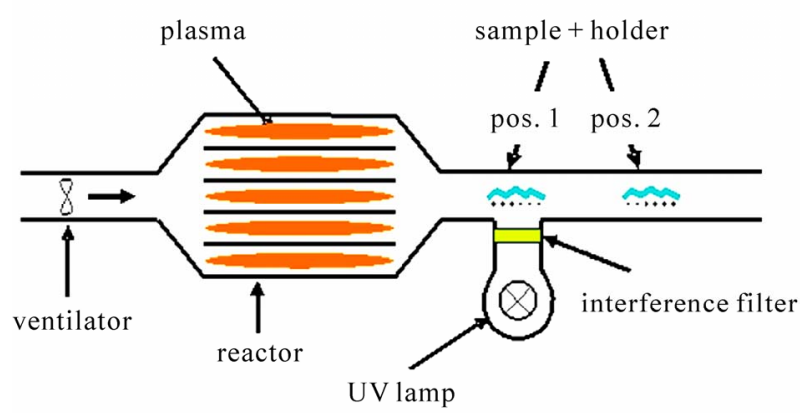

Figure 2. Experimental set up for treatment of textiles by SDDBD and UV-radiation.

\section{Plasma generation Activation of oxygen}
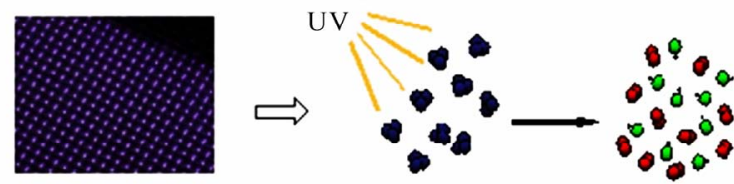

Air

$\mathrm{O}_{3}$ $\mathrm{O}_{2}+\mathrm{O}$

Figure 3. Scheme of the ozone activation by UV radiation. 
level is building up again.

The Figures 5 to 7 present the results of the microbial tests. We carried out different test series with the devices and methods described above to proof the possible application of plasma procedures for the disinfection of textiles.

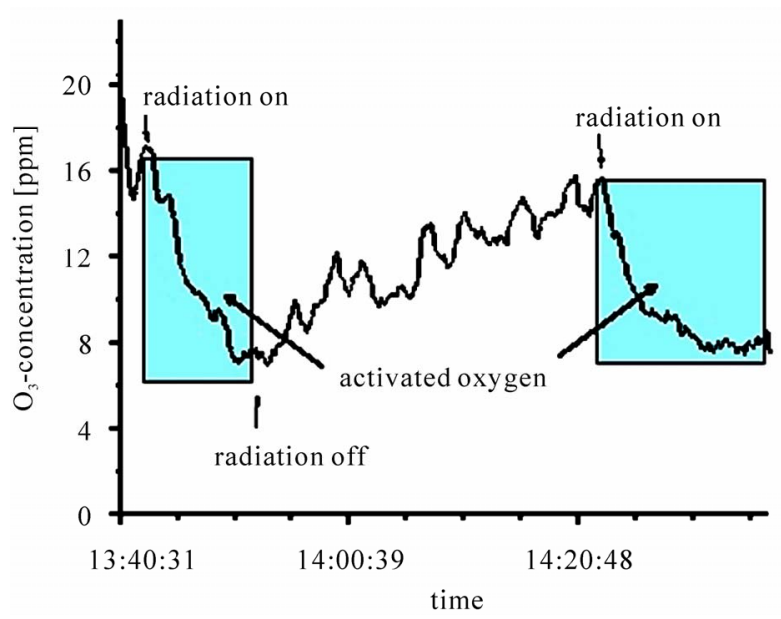

Figure 4. The influence of UV radiation on the ozone concentration.

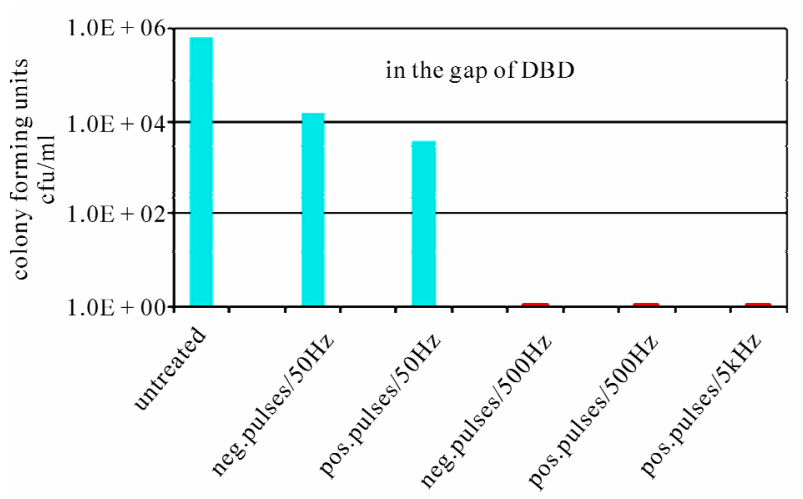

Figure 5. Results of the treatment in the discharge gap of a DBD.

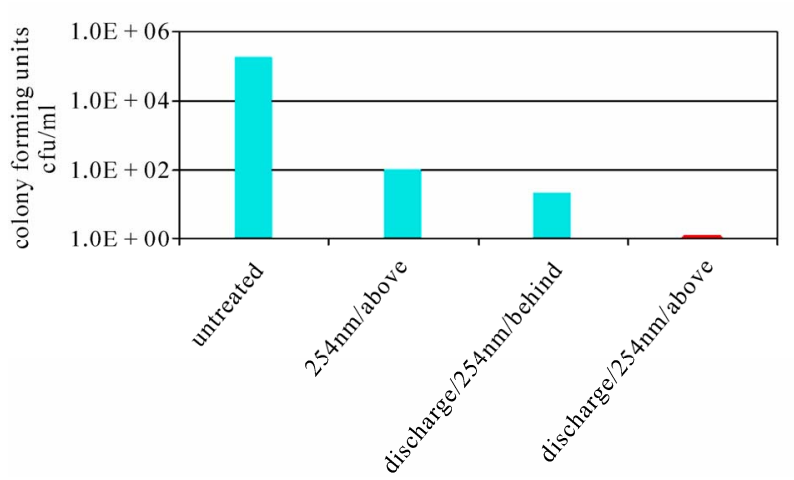

Figure 6. Influence of discharge and ultraviolet radiation.

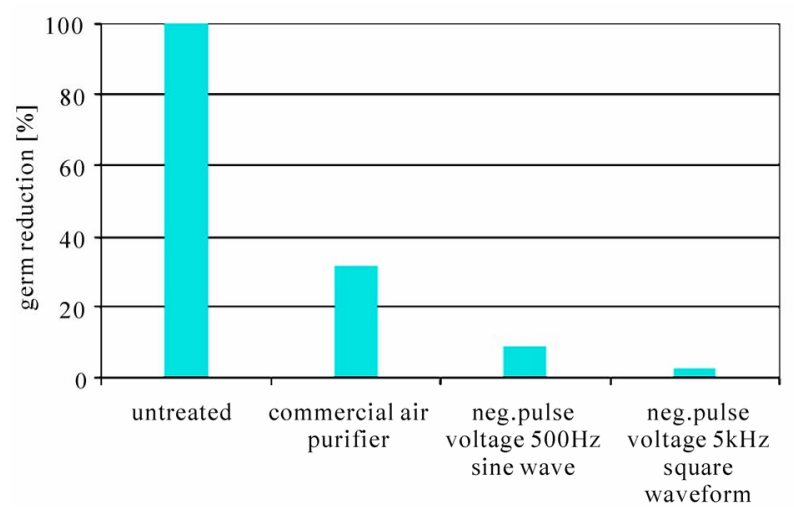

Figure 7. Comparison between ion extraction method and commercial air purifier.

Figure 5 shows results of the treatment in the discharge gap of a DBD with different frequencies, positive and negative pulse voltages of $7.5 \mathrm{kV}$ and a treatment time of 30 minutes.

With the treatment in the discharge gap and a frequency of $50 \mathrm{~Hz}$ about two-log reductions in number of germs is observed. The best results are obtained by using higher frequencies with more than six-log reductions in the number of germs.

Figure 6 shows results when ultraviolet radiation comes into operation. In this case an experimental arrangement has been used such as in Figure 2.

The application of ultraviolet radiation $(254 \mathrm{~nm})$ already causes a reduction of about approx. 3 log 10 steps. When we add additional a DBD a reduction over more than $5 \log$ steps emerges.

Figure 7 shows the effect of the ion extraction method and a commercial air purifier. A commercial air purifier causes a reduction of the germs of about approx. $70 \%$. The according results with the ion extraction methode were performed with different pulse voltages. In the case of negative pulses $91 \%$ and in the case of positive pulse voltages $98 \%$ of germes were reduced.

$\mathrm{dc}$, and rms do not have to be defined. Do not use abbreviations in the title or heads unless they are unavoidable.

\section{CONCLUSIONS}

Atmospheric pressure plasmas possess the ability to reduce smell effectively. In addition a significant reduction of microorganism population in some of the samples could be achieved.

In case of odours it was already shown for the processing gas air loaded with aldehydes or butanone that an oxidation or a decomposition of hydrocarbon compounds may occur [7]. Here these reactions can be also assumed as cleaning mechanisms. 
The sterilization of the textile samples served noticeable results in particular for the procedures with direct plasma contact as well as in case of the oxygen excitation by UV-light. In case of the oxygen excitation we can assume reactions between excited atomic oxygen and germ cell walls.

By direct exposure of germs with plasma a complex and complicated impact of several effects occurs like radiation and reactions of different radicals with the germs. A charge accumulation and rupture of the outer membrane of bacterial cells is also conceivable.

The attempts with the treatment by ions, with commercial ionizer as well as with the introduced ion extraction procedure, show a noticeable effect. This indicates possible ion molecule reactions.

The presented investigations are a first step. For examination of different disinfection mechanisms further systematic investigations are necessary. In particular the time dependence of the mechanisms is an important matter.

The relatively simple designs of proposed DBD configurations and methods give them the potential to replace conventional cleaning methods in described application fields. It could help to save water consumption and to develop a cleaning procedure of textiles without the use of detergents.

\section{ACKNOWLEDGEMENTS}

The authors are grateful to Kirsten Anklam, Diana Neudeck, Tila Krüger and Norman Mleczko for their technical support.

\section{REFERENCES}

[1] Penetrante, B.M. and Schultheis, S.E. (1993) NATO advanced research workshop on non-thermal plasma techniques for pollution control. NATO ASI Series G:
Ecological Sciences, Springer-Verlag Berlin-Heidelberg.

[2] Kim, H.-H. (2004) Plasma Process. Polym., (1), 91-110.

[3] Grundmann, J., Müller, S. and Zahn, R.-J. (2005) Plasma Chem. Plasma Process., (25), 455-456.

[4] Müller, S., Conrads, J. and Best, W. (2000) Int. Sympos. on High Pressure Low Temperature Plasma Chem., Contributed Papers, (2), 340-344.

[5] Kogelschatz, U. (2007) Plasma Process. Polym. (4), 678-681.

[6] Becker, K.H., Kogelschatz, U., Schoenbach, K.H. and Barker, R.J. (2005) Non equilibrium air plasmas at atmospheric pressure. Inst. of Phys. Publ., IOP Publishing Ltd, Bristol and Philadelphia.

[7] Müller, S. and Zahn, R.-J. (2007) Contr. to Plasma Phys. (47), 520-529.

[8] Mendis, D.A., Rosenberg, M. and Azam, F. (2000) IEEE Trans. Plasma Sci. (28), 1304-1306.

[9] Laroussi, M., Richardson, J.P. and Dobbs, F.C. (2002) Appl. Phys. Lett. (81), 772-774.

[10] Stoffels, E., Flikweert, A.J., Stoffels, W.W. and Kroesen, G.M.W. (2002) Plasma Sources Sci. Technol. (11) 383-388.

[11] Laroussi, M., Mendis, D.A. and Rosenberg, M. (2003) New Journ. Phys. (5) 41.1-41.10.

[12] Laroussi, M. (2005) Plasma Process. Polym. (2) 391-400.

[13] Laroussi, M., Tendero, C., Lu, X., Alla, S. and Hynes, W.L. (2005) Plasma Process. Polym. (3), 470-473.

[14] Brandenburg, R., Ehlbeck, J., Stieber, M., Woedtke, T.v. Zeymer, J., Schlüter, O. and Weltmann, K.-D. (2007) Contr. to Plasma Phys. (47), 72-79.

[15] Vicoveanu, D., Ohtsu, Y. and Fujita, H. (2008) Jap. Journ. of Appl. Phys. (47), 1130-1135.

[16] Vicoveanu, D., Popescu, S., Ohtsu, Y. and Fujita, H. (2008) Plasma Process. Polym. (5), 350-358.

[17] Kang, J.Y. and Samardi, M. (2004) AATCC Review (10), 28-32.

[18] Shishoo, R. (2007) Plasma technologies for textiles. Woodhead Publishing Limited, Cambridge, UK, Woodhead Textiles Series No. 62.

[19] Müller, S., Zahn, R.-J. and Grundmann, J. (2007) Plasma Process. Polym. (4), S1004-S1008. 